\title{
Vagus Nerve Schwannoma At Cerebellopontine Angle With Extracranial Extension Through Jugular Foramen
}

Hao Tang ( $\square$ tanghao6251@163.com )

PLA General Hospital Hainan Bra https://orcid.org/0000-0003-2435-7293

\section{Zhenghui Sun}

PLA General Hospital Hainan Bra

Jiashu Zhang

Chinese PLA General Hospital

Xinguang $\mathrm{Yu}$

Chinese PLA General Hospital

\section{Case report}

Keywords: Vagus Nerve, Jugular Foramen, Parapharyngeal space, Schwannoma

Posted Date: August 5th, 2021

DOI: https://doi.org/10.21203/rs.3.rs-763030/v1

License: (c) (i) This work is licensed under a Creative Commons Attribution 4.0 International License. Read Full License 


\section{Abstract}

Communicating tumors between the posterior fossa and parapharyngeal space are rare and difficult to diagnose and treat. Surgical excision of these space lesions is challenging because of the anatomical complexity of the area. In this case we successfully made full resection of a big rare vagus nerve schwannoma at cerebellopontine angle and parapharyngeal space. Surgical resection is the treatment of first choice for this disease. The degree of lesion adhesion to the brain stem and peripheral vessels determines the degree of tumor resection and prognosis of patients.

\section{Introduction}

Communicating tumors between the posterior fossa and parapharyngeal space are rare and difficult to diagnose and treat. These tumors have different histological origins such as schwannomas, neurofibromas, paragangliomas and salivary gland tumors. Most of these lesions are benign but $20 \%$ have malignant transformation ${ }^{[1]}$. These tumors may remain undetected for long periods of time and usually remain asymptomatic until they become large enough to displace and compress nearby structures. Surgical excision of tumors from the parapharyngeal space is difficult due to the complex anatomy of the region which contains numerous neurovascular structures, as well as the difficult surgical approach to the area ${ }^{[2]}$.

In this case we successfully made full resection of a big rare vagus nerve schwannoma at cerebellopontine angle with extracranial extension through Jugular Foramen. During operation, we confirmed the tumor was neurinoma originate from posterior cranial nerve. The prognosis of schwannoma after complete excision is good and recurrence is not happened during 2years follow-up.

\section{Presentation Of Case}

Clinical Presentation. A 54-year-old female was referred to our institution with an intermittent headache for more than 7 years and 2 weeks of aggravation. Subcutaneous palpable lumps were found in the left neck, no obvious tenderness, no wave motion, and tight connection with surrounding tissues. She did not demonstrate any other neurological deficits. Neurological examination revealed that he had no hearing loss no positive Romberg sign.

Investigation and Radiological Aspects. CT scanning shows an occupying lesion at left cerebellopontine angle and no enlargement of internal auditory canal but enlargement of left jugular foramen. MRI shows a cystic-solid occupying lesion at left cerebellopontine angle and extracranial extension to left parapharyngeal space. MRA and MRV shows nothing unnormal of intercranial vessels. Besides, DSA shows abundant blood-supply of tumor mainly from left vertebral artery and partly left ECA (external carotid artery). After evaluation, embolization therapy was rejected since its high risk. (Fig. 1.) MRI scanning shows in the left cerebellopontine angle area, irregular, slightly long / long T1, short / long T2 mixed signals were observed. The lesion extends downward along the left jugular foramen and the carotid 
sheath area, and the DWI of part lesion is slightly hyperintense. After enhancement, there is an obvious inhomogeneous flake and ring strengthening, the brain stem and the four ventricle is slightly shifted to the right. (Fig. 2.)

Differential Diagnosis. Glioma, ependymoma, medulloblastoma and meningioma at foramen magnum were considered. But since the lesion had clear boundary, no obvious edema, no dural tail sign, neurilemmoma is still the first consideration.

Operation and Pathological Findings. The patient underwent tumor resection via a suboccipital extreme lateral approach. After the musculocutaneous flap was turned around, the occipital scale, foramen magnum, posterior arch of atlas and vertebral artery were exposed. The tissue around the internal jugular vein was exposed layer by layer, and the left posterior arch of atlas was bitten off to the notch of vertebral artery. After craniotomy of the occipital squama and retrosigmoid bone flap, the edge of sigmoid sinus was exposed laterally, part of occipital condyle was removed, mastoid process was further removed and jugular foramen was opened. During operation, after opening jugular foramen, tumor can be seen grew into and out of the cranium respectively. Microscopically, the tumor is a neurilemmoma arising from one of the posterior cranial nerves. The tumor was yellow in color, soft in texture, rich in blood supply, with obvious capsule. The tumor sheath was cut to expose the tumor under microscope. Tumor was separated along the course of the tumor to the extracranial part of the neck. Extracranial tumors are supplied by the branches of the external carotid artery. The left internal jugular vein and jugular bulb have been oppressed to occlusion by the tumor. Extracranial tumor got total excision after devascularization. The residual nerve ends were found after the tumor was removed (Fig. 3.A). After further incision of the dura and retraction of left cerebellar hemisphere, intracranial tumors were found located in cerebellopontine angle and cisterna magna. Facial nerve, trigeminal and abducens nerve are compressed upward by tumor. There was no obvious adhesion between the tumor and the brainstem, facial auditory nerve, abducent nerve and trigeminal nerve. Part of the tumor had tight adhesion with the pontine. (Fig. 3.B) The tumor was removed piece by piece and got total resection under microscope. Pathological examination revealed schwannoma with cystic degeneration, which confirmed the histological diagnosis. (Fig. 4.)

Postoperative Course. The patient consciousness is completely restored 3days after operation, and the endotracheal tube was removed then. The operation did not cause any cerebrospinal fluid leakage, even in the early postoperative period. After operation, the patient had mild facial paralysis and high heart rate for a few days since the tumor was adjacent to the brainstem, but headache disappeared. The patient had tracheotomy 5 days after operation since poor expectoration ability. 3 months after operation, the patient has recovered well, and fellow-up MRI study showed no any remnant or recurrence of the tumor. (Fig. 5.ABC) 2 years follow-up MRI also showed no any remnant or recurrence of the tumor. (Fig. 5.DEF)

\section{Discussion}

The vagus nerve is cranial nerve $X$, which origin from the medulla under glossopharyngeal nerve root and through jugular foramen out of cranium. Vagal schwannoma is a benign, slow-growing mass, often 
asymptomatic, with a very low lifetime risk of malignant transformation in general population, but diagnosis is still a challenge ${ }^{[3]}$. The common tumors in the CPA region including: acoustic neuroma, meningioma, trigeminal neurinoma, ependymoma, glomus jugular tumor, papilloma choroideum and so on. In this case, differential diagnosis is not very difficult. Based on CT and MRI, neurinoma is first consideration, and it's not likely from acoustic nerve nor trigeminal nerve as well. During operation, we confirmed the tumor was neurinoma originate from posterior cranial nerve. The combination of CT, MRI and MRS studies are very helpful in preoperative diagnosis of CPA area lesions. Vagus nerve schwannoma at cerebellopontine angle is quite rare besides such a huge lesion (about $5 \mathrm{~cm} \times 4.5 \mathrm{~cm}$ ) grow through jugular foramen to poststyloid parapharyngeal space ${ }^{[2,4]}$.

Surgical resection is the treatment of first choice even if its close relationship with nerve fibers, from which it arises, threats vagal nerve preservation ${ }^{[5,6]}$. The size of the tumor is directly related to the risk of surgery ${ }^{[7]}$. Preoperative preparation of blood and intraoperative electrophysiological monitoring are necessary methods to reduce risk and preserve facial and acoustic nerve. The degree of lesion adhesion to the brain stem and peripheral vessels determines the degree of tumor resection ${ }^{[8]}$. Fortunately, in this case the adhesion is not very severe, and lesion has got complete excision finally. In the 9 months followup, no remnant or recurrence of the tumor was observed.

The patient regained consciousness on third day after operation and had a fast heart rate since the lesion so close to brain stem. At recovery phase, the patient had tracheotomy on the sixed day after operation and with tracheal intubation for 2 months. Now the patient is recovered and can pronounce most of the words although with hoarseness. The patient has no facial paralysis nor hearing loss after operation since good neural protection during operation. But she has dysphagia after operation because of the resection of vagus nerve and she need gastric tube to eating since then.

\section{Conclusions}

In this case we successfully made full resection of a big rare vagus nerve schwannoma at cerebellopontine angle and parapharyngeal space. Surgical resection is the treatment of first choice for this disease. The degree of lesion adhesion to the brain stem and peripheral vessels determines the degree of tumor resection.

\section{Declarations}

Ethics approval and consent to participate

Ethical approval to report this case series was obtained from Hainan Hospital Ethics Committee of PLA General Hospital.

Verbal informed consent was obtained from a legally authorized representative(s) for anonymized patient information to be published in this article. 
Consent for publication

All presentations of this case report have got consent for publication from patient and her family.

Availability of data and materials

The datasets during and/or analysed during the current study available from the corresponding author on reasonable request.

Competing interests

The authors declare that they have no competing interests.

Funding

This research received no specific grant from any funding agency in the public, commercial, or not-forprofit sectors.

Authors' contributions

TH analyzed and interpreted the patient data, write the paper, and was a major contributor in writing the manuscript. TH, ZJS and SZH contribute to the operation and YXG Gave guidance to this paper. All authors read and approved the final manuscript.

Acknowledgements

Not applicable

\section{References}

1. Katre M, Telang R. Schwannoma of parapharyngeal space: a case report. Indian J Surg. 2013;77(1):79-81.

2. Carroll $C$, Jagatiya $M$, Kamel $D$, et al. A parapharyngeal space schwannoma arising from the vagus nerve: A case report. International Journal of Surgery Case Reports. 2017;41:22-5.

3. Kamath PM, Dosemane D, Sreedharan SS, Majeed NA, Shenoy VS. Vagal Schwannoma: A Rare Parapharyngeal Tumour. J Clin Diagn Res. 2016 Apr;10(4):MD03-4.

4. Chiun KC, Tang IP, Prepageran N, Jayalakshmi P. An extensive cervical vagal nerve schwannoma: a case report. Med J Malaysia. 2012 Jun;67(3):342-4.

5. Abdulla FA, Sasi MP. Schwannomatosis of Cervical Vagus Nerve. Case Rep Surg, 2016, 2016:8020919.

6. Behuria S, Rout T, Pattanayak S. Diagnosis and management of schwannomas originating from the cervical vagus nerve. Ann R Coll Surg Engl. 2015;97(2):92-7. 
7. Ramdass AA, Yao M, Natarajan S, et al. A Rare Case of Vagus Nerve Schwannoma Presenting as a Neck Mass. American Journal of Case Reports. 2017;18:908-11.

8. Cavallaro G, Pattaro G, lorio O, Avallone M, Silecchia G. A literature review on surgery for cervical vagal schwannomas. World J Surg Oncol. 2015 Mar;29:13:130.

\section{Figures}
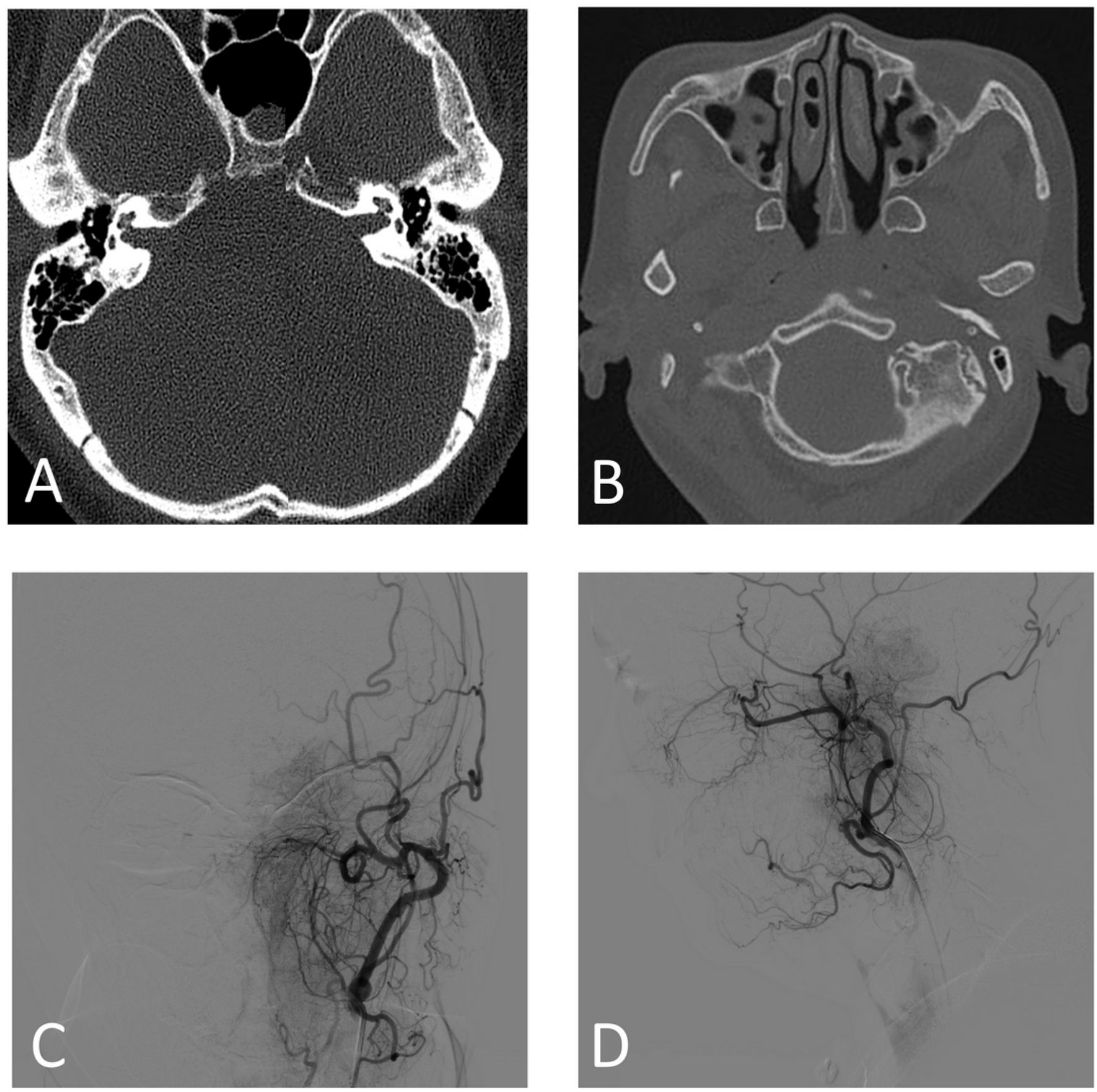

Figure 1 
Preoperative images of differential diagnosis. A-B: Axial CT scan showing no enlargement or erosion of the internal auditory canal(A), but enlargement of left jugular foramen(B). C-D: Positive and side view of digital subtraction angiography of the lesion showing it with abundant blood supply mainly from ECA and posterior inferior cerebellar artery.
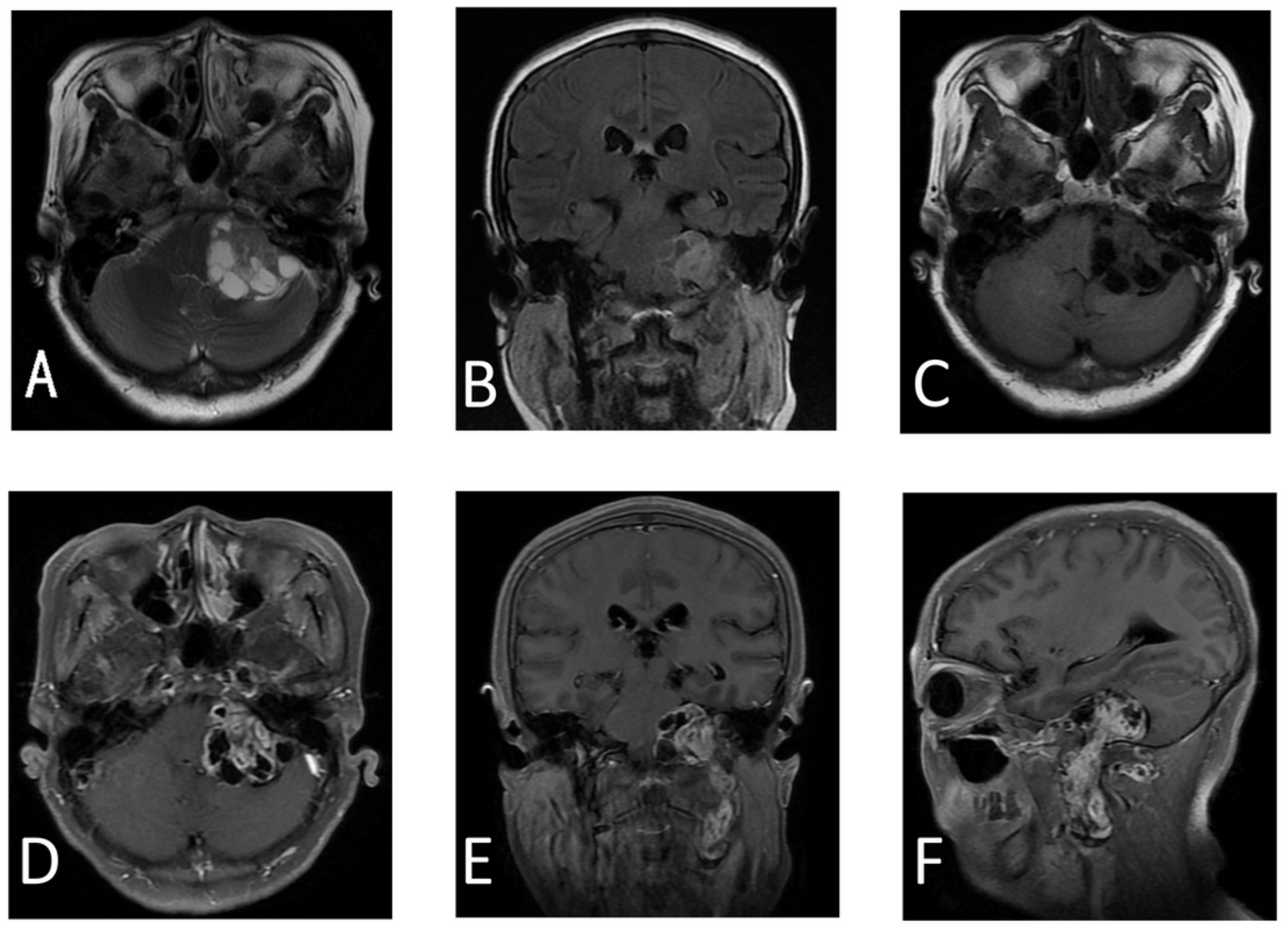

\section{Figure 2}

Preoperative images of the lesion. A: Axial T2 MRI. B: Coronal T2 Flair MR image. C: Axial T1 Flair MR image. D-F: Axial, Coronal, and Sagittal Gd-enhanced MR images showing the lesion extending from the tentorium downward to the left para-pharyngeal space through jugular foramen with inhomogeneous enhancement. 

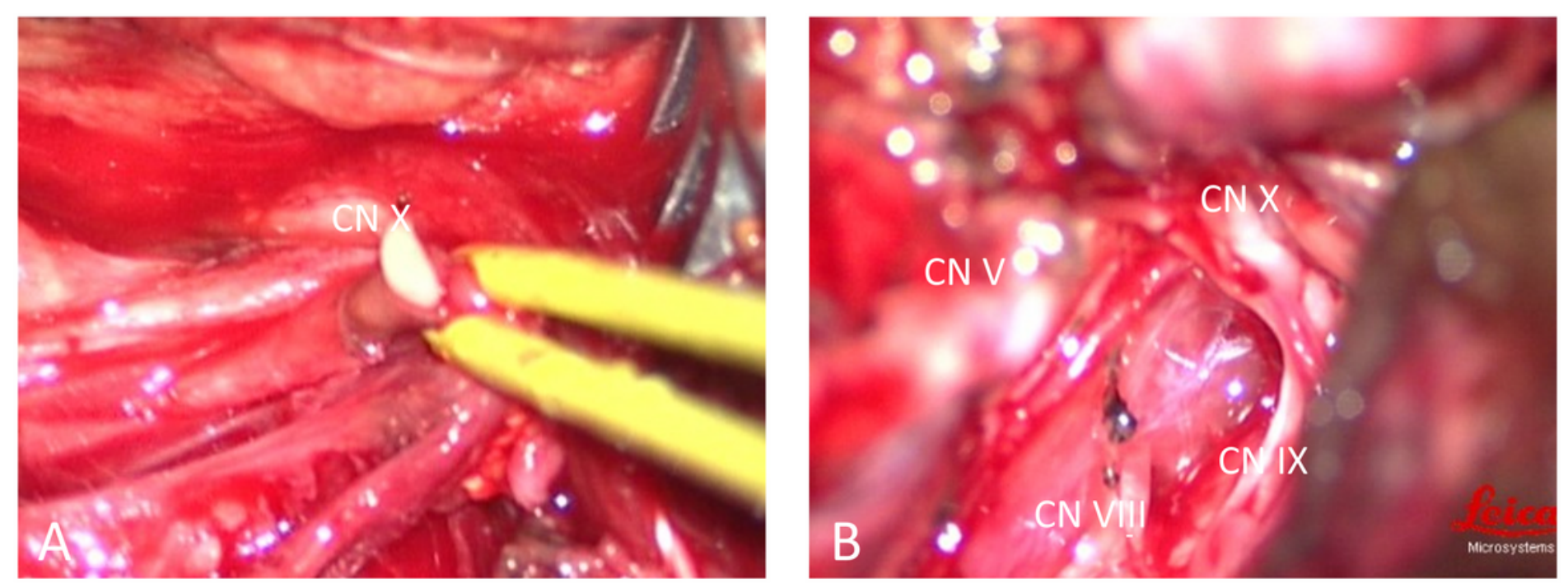

\section{Figure 3}

Intraoperative images. A: Extracranial lesions were cut off and the broken end of Central Nerve X. B: Intracranial lesions were totally removed, and surrounding structures remained intact after resection. (3) 


\section{Figure 4}

Histopathological examination with H\&E staining revealed the lesion is full of densed spindle cell tumor. Original magnification $\times 100$.
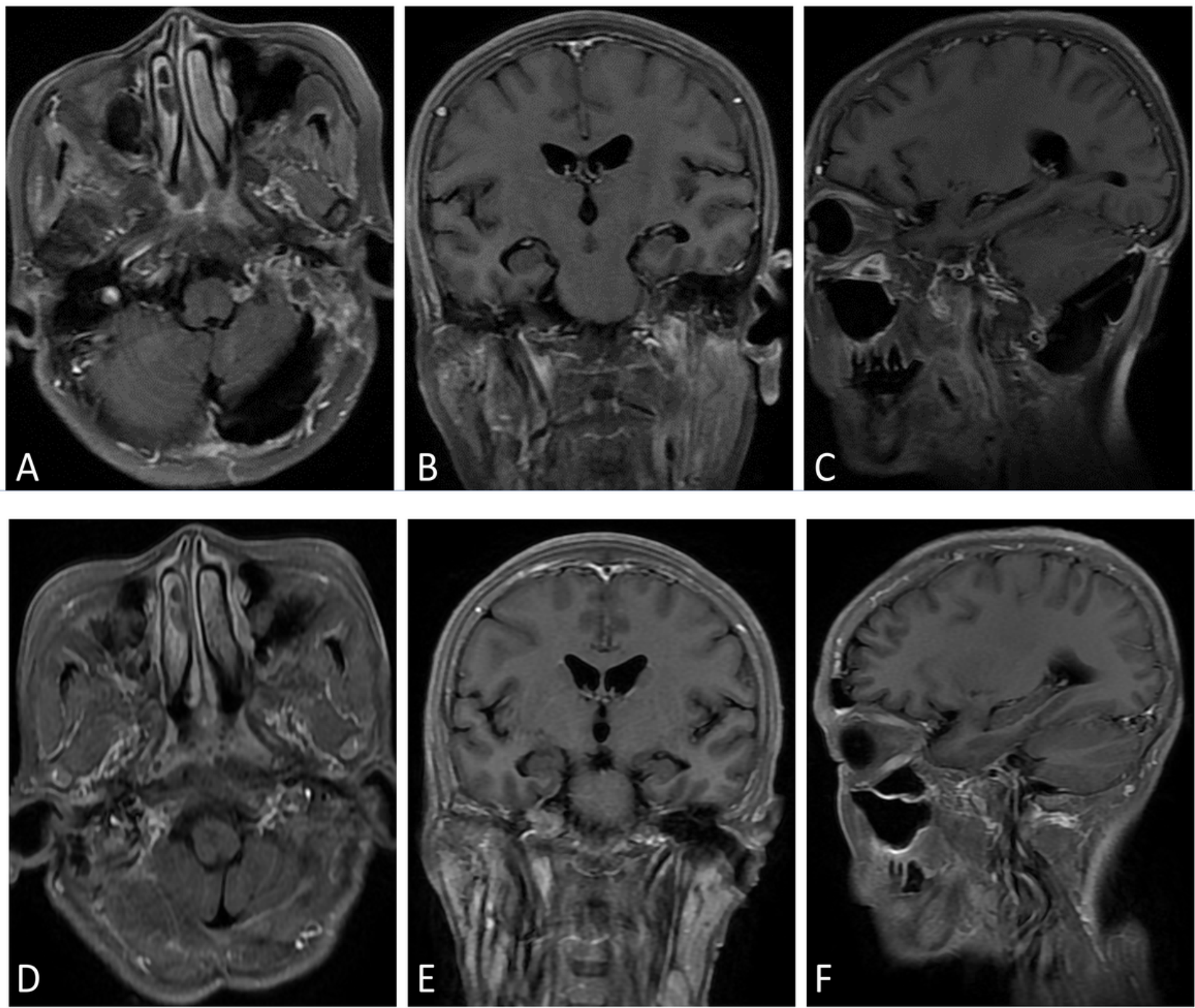

\section{Figure 5}

Axial, coronal and sagittal Gd-enhanced MR images of fellow-up MRI at 3 months (A-C) and 26 months (D-F) after surgery showed no tumor recurrence 